\title{
Validación inicial de una escala para medir el nivel de sobrecarga de padres o cuidadores de niños asmáticos
}

\author{
Ángela M. Pedraza ${ }^{1}$, Carlos E. Rodríguez-Martínez ${ }^{2,3,4}$, Ranniery Acuña ${ }^{4}$ \\ 1 Departamento de Neumología Pediátrica, Hospital Universitario San Rafael, Bogotá, D.C., Colombia \\ 2 Departamento de Pediatría, Facultad de Medicina, Universidad Nacional de Colombia, Bogotá, D.C., Colombia \\ ${ }^{3}$ Departamento de Neumología Pediátrica y Cuidado Intensivo Pediátrico, Universidad El Bosque, Bogotá, D.C., \\ Colombia \\ ${ }^{4}$ Subdirección de Docencia e Investigación Científica, Hospital Militar Central, Bogotá, D.C., Colombia
}

Introducción. A pesar de la alta probabilidad de que los padres o cuidadores de niños asmáticos presenten sobrecarga, no se han validado instrumentos para medirla en el contexto del asma pediátrica.

Objetivo. Iniciar la validación de una escala para medir la sobrecarga de los padres o cuidadores de niños asmáticos y determinar los factores asociados con esta sobrecarga.

Materiales y métodos. Mediante un estudio analítico de corte transversal se midió el nivel de sobrecarga de los padres o cuidadores de niños asmáticos de 1 a 16 años, utilizando una versión abreviada de la escala de Zarit. Se hizo un análisis factorial exploratorio de los componentes principales de esta escala, y se evaluó su validez de constructo y su validez interna. Se ajustaron modelos de regresión logística para identificar los factores asociados con una sobrecarga intensa en los padres o cuidadores de los niños asmáticos.

Resultados. La mediana (rango intercuartílico) de la edad de los 162 niños analizados fue de 6 años (rango, 4 a 9). Del total de padres o cuidadores, 37 (22,8 \%) estaban experimentando sobrecarga intensa. El análisis factorial mostró una estructura probable de dos factores que, en conjunto, explican el $61,5 \%$ de la varianza total de los resultados. La única variable que se asoció de forma independiente con una sobrecarga intensa en el análisis multivariado, fue el asma no controlada $\left(\mathrm{OR}=5,38 ; \mathrm{IC}_{95}\right.$ : 1,43-20,16; $p=0,012)$.

Conclusiones. La escala de Zarit utilizada tiene una aceptable validez interna y una adecuada validez de constructo para determinar el nivel de sobrecarga que experimentan los padres o cuidadores de niños asmáticos. El grado de control del asma del niño fue la única variable que se asoció de forma independiente con este nivel de sobrecarga.

Palabras clave: asma, estudios de validación, carga de trabajo, padres, niños, cuidadores, pediatría. doi: http://dx.doi.org/10.7705/biomedica.v33i3.813

Initial validation of a scale to measure the burden for parents/caregivers of children with asthma and factors associated with this burden in a population of asthmatic children

Introduction. Despite the high probability of a heavy burden for parents and/or caregivers of children with asthma, instruments intended to measure it have not been validated in the context of asthma. Moreover, factors associated with this burden are unknown.

Objective. To begin the validation of a scale to measure the burden for parents/caregivers of asthmatic children, and to determine the factors associated with this burden.

Materials and methods. We performed an analytical cross-sectional and validation of scale study. At the outpatient section of the Clinic of Pediatric Pulmonology in the Hospital Universitario Clinica San Rafael, we measured the burden of parents and/or caregivers of children with asthma using a shortened version of the Zarit scale throughout 2011. We performed a principal components factor analysis and adjusted logistic regression models to identify independent factors associated with a heavy burden.

Results. The median (interquartile range) age of the 162 children included in the study was 6 (4-9) years. Out of the total of parents and/or caregivers, 37 (22.8\%) were experiencing a heavy burden. Factor analysis of the Zarit scale showed a probable structure of two factors, which together accounted for $61.5 \%$ of the variance of the results. Uncontrolled asthma was the only predictor independently

\section{Contribución de los autores:}

Ángela M. Pedraza ideó el estudio, escribió el protocolo de investigación, recolectó los datos y escribió la sección de discusión. Carlos E. Rodríguez-Martínez hizo el análisis estadístico, interpretó los datos y escribió la sección de metodología y resultados. Ranniery Acuña escribió el protocolo de investigación, recolectó los datos, y escribió las secciones de introducción y discusión. 
associated with a heavy burden in the multivariate analysis (OR 5.38; 95\% $\mathrm{Cl} 1.43-20.16, \mathrm{p}=0.012$ ).

Conclusions. The Zarit scale used has an adequate factorial structure, an acceptable internal consistency, and an adequate construct validity for measuring the burden for parents and/or caregivers of children with asthma. The level of a child's asthma control is the most important independent predictor of this burden.

Key words: asthma, validation studies, workload, parents, children, family caregivers, pediatrics doi: http://dx.doi.org/10.7705/biomedica.v33i3.813

El asma es la enfermedad crónica más frecuente de la niñez, con una prevalencia puntual estimada en Colombia de alrededor de $10 \%(1-3)$, con un aparente aumento en los últimos 10 años (4), que se considera un problema de salud pública en nuestro país. A pesar de los importantes descubrimientos recientes en la fisiopatología del asma y de la disponibilidad de opciones terapéuticas muy efectivas, aún hoy en día el asma genera una importante morbilidad en niños, con un impacto negativo en la calidad de vida, no sólo de los niños que la padecen, sino también en la de sus padres o cuidadores (5).

Se define como sobrecarga de cuidadores al grado en que la persona encargada de cuidar percibe que las diferentes esferas de su vida (proyecto vital, vida social, relaciones familiares, tiempo libre, desatención de la propia salud, privacidad) se afectan por dicha labor (6). Las repercusiones en la salud física y mental que pueden llegar a presentar los cuidadores, han dado origen al denominado "síndrome del cuidador" (7). Se ha descrito que, en comparación con los cuidadores sin sobrecarga, aquellos que experimentan sobrecarga pueden presentar con mayor frecuencia trastornos de ansiedad y depresión $(8,9)$, mayor aislamiento social, deterioro de la situación económica familiar $(10,11)$, mayor morbilidad general (12) e, incluso, mortalidad (13), lo cual puede no sólo tener repercusiones en la salud y el bienestar físico y emocional del cuidador mismo, sino también en la persona que está bajo su cuidado (14).

A pesar de que la expresión "sobrecarga de cuidadores" se ha utilizado más en personas que tienen a su cargo el cuidado de ancianos que padecen enfermedades degenerativas, también se ha descrito en personas que cuidan a niños con discapacidad, habiéndose descrito fatiga,

\section{Correspondencia:}

Carlos E. Rodríguez Martínez, Avenida calle 127 № 20-78,

Bogotá, D.C., Colombia

Teléfono: (571) 259 5500; fax: (571) 2595520

carerodriguezmar@unal.edu.co

Recibido: 27/08/12; aceptado:18/03/13 depresión, baja autoestima e insatisfacción interpersonal en estos cuidadores (15). En lo que se refiere específicamente a niños asmáticos, se han descrito importantes alteraciones en la calidad de vida de sus padres o cuidadores (16), relacionadas principalmente con ciertos factores sociodemográficos, con la frecuencia e intensidad de los síntomas, con la gravedad de la enfermedad, y con la efectividad de los medicamentos para su tratamiento (17-19). Debido a la alta prevalencia de asma en la población pediátrica y a las afectaciones emocionales y la limitación de actividades que se ha descrito que presentan los padres o cuidadores de estos niños (10), es concebible considerar que la enfermedad genere una significativa sobrecarga en las personas que tienen a su cargo a estos pacientes.

Sin embargo, a pesar de que el concepto "calidad de vida" comprende varios dominios que pueden estar relacionados con la sobrecarga, tales como bienestar emocional, relaciones interpersonales, bienestar material, desarrollo personal, bienestar físico, autodeterminación e inclusión social (20), hasta donde sabemos, la sobrecarga de los padres o cuidadores de niños asmáticos no se ha medido directamente. De igual forma, no se han determinado los factores asociados con esta sobrecarga.

El objetivo del presente estudio fue hacer la validación inicial de una versión abreviada de la escala para medir la sobrecarga del cuidador desarrollada por Zarit (21), y determinar los factores asociados con el nivel de sobrecarga de los padres o cuidadores en una población de niños asmáticos.

\section{Materiales y métodos}

Se hizo un estudio analítico de corte transversal y de validación de escala. Mediante un muestreo no probabilístico secuencial por conveniencia, se invitó a participar en el estudio a los padres o cuidadores de los pacientes con diagnóstico clínico de asma, con edades comprendidas entre 1 y 16 años, que asistieron al Servicio de Consulta Externa de Neumología Pediátrica del Hospital Universitario 
Clínica San Rafael entre el $1^{\circ}$ de enero y el 31 de diciembre de 2011.

Los padres o cuidadores que aceptaron participar en el estudio y que firmaron el consentimiento informado, completaron un cuestionario que incluyó información relacionada con el niño asmático (edad, sexo, grado de control de asma) y con datos generales de los padres o cuidadores (edad, escolaridad, estado civil y clase de cuidador principal) y con el nivel de sobrecarga del padre o cuidador.

El grado de control del asma se valoró según los parámetros recomendados en las guías de la Global Initiative for Asthma (GINA) (22), y se clasificó como no controlada, parcialmente controlada y controlada.

\section{Escala de sobrecarga del cuidador}

Para medir el nivel de sobrecarga del cuidador se utilizó una versión abreviada de la "Escala de sobrecarga del cuidador de Zarit" (6). Esta escala es el instrumento más utilizado internacionalmente para medir la sobrecarga de cuidadores, debido a las múltiples ventajas que tiene con respecto a otras escalas que intentan objetivar la carga del cuidador.

Se ha considerado que la escala de Zarit abarca todos las dimensiones del síndrome del cuidador y ha sido validada en varios idiomas, incluyendo el español $(23,24)$. Ha mostrado ser transculturalmente válida en Colombia (25), y tener una adecuada validez de apariencia, de contenido y de constructo en diferentes adaptaciones lingüísticas $(26,27)$. Además, tanto en su estudio original como en sus validaciones internacionales, brinda una gran confiabilidad entre observadores (con valores de coeficiente de correlación intraclase que varían entre 0,71 y 0,85$)(6,26,28)$ y una adecuada validez interna (con valores de alfa de Cronbach que varían entre 0,85 y 0,93$)(6,23,26)$.

La versión original en inglés, que tiene derechos de autor desde 1983, consta de 22 preguntas con opciones de respuesta con una escala de tipo Likert de cinco puntos, con un rango que va desde 1 (nunca) hasta 5 (casi siempre); sus puntuaciones suman un total de 22 a 110 puntos y, entre más altas, indican mayor sobrecarga. Con base en la puntuación total de la escala, se han establecido dos umbrales para diagnosticar: ausencia de sobrecarga, con un puntaje menor o igual a 46, sobrecarga ligera, con uno de 47 a 55 , y sobrecarga intensa, con uno de 56 o más.
Según Gort, et al., en las diferentes preguntas de la escala de Zarit se exploran diversos campos: la sensación de sobrecarga experimentada por el cuidador, el abandono del autocuidado tanto en temas de salud como en autoimagen, la vergüenza ante la presencia o comportamiento del familiar enfermo, la irritabilidad ante la presencia del familiar, el miedo por los cuidados o el futuro del familiar, la pérdida de rol social y familiar al tener que asumir el de cuidador, la alteración de la economía y el sentimiento de culpabilidad generado por no hacer lo suficiente (21). Sin embargo, Ankri, et al., mediante el uso de un análisis factorial exploratorio determinó sólo tres dominios de utilidad para los médicos tratantes que enfrentan a estos cuidadores: efectos en la vida personal y social de los cuidadores, carga psicológica y sentimientos de culpa (24).

Las principales desventajas de la escala de Zarit son su extensión y el tiempo requerido para contestar todas las 22 preguntas, lo cual puede ser una carga más para un cuidador con ya múltiples funciones y ocupaciones a su cargo. Por este motivo, en los últimos años se han usado diferentes versiones abreviadas de la escala de Zarit, de las cuales, la versión en español utilizada y validada por Regueiro, et al., y Gort, et al. $(21,29)$, es una de las más utilizadas.

Esta escala consta de sólo siete preguntas de la original y al igual que ella, tiene opciones de respuesta con una escala de tipo Likert de cinco puntos, con un rango que va desde 1 (nunca) hasta 5 (casi siempre); sus puntuaciones suman un total de 75 a 35 puntos y, entre más altas, indican mayor sobrecarga (cuadro 1). La escala abreviada ha mostrado disminuir el tiempo requerido para completarla, optimizando el tiempo del cuidador y el del médico tratante. Además, al compararla con la escala original como método de referencia y tomando 17 como punto de corte para diferenciar a los cuidadores con sobrecarga intensa de aquellos sin sobrecarga intensa, mostró tener una sensibilidad de $100 \%$, una especificidad de $90,5 \%$, un valor pronóstico positivo de $95,5 \%$ y un valor pronóstico negativo del $100 \%$.

Debido a estas propiedades y al menor tiempo para completar la versión abreviada, se decidió utilizarla en el presente estudio. Sin embargo, debido a que hasta donde sabemos esta escala abreviada no ha sido nunca utilizada para medir el nivel de sobrecarga de padres o cuidadores de niños asmáticos y a que solo se ha descrito la 
Cuadro 1. Escala abreviada de Zarit

1. ¿Siente usted que, a causa del tiempo que gasta con su familiar/paciente, ya no tiene tiempo suficiente para usted mismo?
Nunca
Casi nunca
A veces
Frecuentemente
Casi siempre
2
3
4
5

2. ¿Se siente estresada(o) al tener que cuidar a su familiar/paciente y tener además que atender otras responsabilidades? (por ejemplo, con su familia o en el trabajo)
Nunca
Casi nunca
A veces Frecuentemente
Casi siempre
2
45

3. ¿Cree que la situación actual afecta a su relación con amigos u otros miembros de su familia de una forma negativa? Nunca Casi nunca Aveces Frecuentemente Casi siempre

$\begin{array}{lllll}1 & 2 & 3 & 4 & 5\end{array}$

4. ¿Se siente agotada(o) cuando tiene que estar junto a su familiar/paciente?

Nunca Casi nunca A veces Frecuentemente Casi siempre

$\begin{array}{rrrrr}1 & 2 & 3 & 4 & 5\end{array}$

5. ¿Siente usted que su salud se ha visto afectada por tener que cuidar a su familiar/paciente? Nunca Casi nunca A veces Frecuentemente Casi siempre

$\begin{array}{lllll}1 & 2 & 3 & 4 & 5\end{array}$

6. ¿Siente que ha perdido el control sobre su vida desde que la enfermedad de su familiar/paciente se manifestó? $\begin{array}{ccccc}\text { Nunca } & \text { Casi nunca } & \text { A veces } & \text { Frecuentemente } & \text { Casi siempre } \\ 1 & 2 & 3 & 4 & 5\end{array}$

7. En general, ¿se siente muy sobrecargada(o) al tener que cuidar de su familiar/paciente?
Nunca
Casi nunca
A veces
Frecuentemente
Casi siempre

2

3

4

5

estructura factorial de la original, pero no de esta escala abreviada, se decidió hacerle un proceso de validación inicial, valorando su validez de constructo y haciendo un análisis factorial exploratorio.

El estudio fue aprobado por el Comité de Ética e Investigación del Hospital Universitario Clínica San Rafael.

\section{Análisis estadístico}

Las variables continuas se presentan como promedios (desviación estándar) o como mediana (rango intercuartílico), según sea apropiado. Las variables categóricas se presentan como porcentajes. Las asociaciones entre las variables categóricas con la variable resultado (sobrecarga intensa Vs. sin sobrecarga intensa) se valoraron con la prueba de ji al cuadrado o el test exacto de Fisher, según fuera apropiado. Las diferencias de las variables continuas entre los cuidadores con sobrecarga intensa y sin ella, se valoraron con la prueba t de Student para muestras independientes o la prueba U de Mann-Whitney, según fuera apropiado.

Se ajustaron modelos de regresión logística para identificar variables asociadas de forma independiente respecto a la presencia de sobrecarga intensa. La fuerza de las asociaciones se evaluó mediante el uso de la razón de momios (odds ratio, $\mathrm{OR}$ ) con sus respectivos intervalos de confianza del $95 \%\left(\mathrm{IC}_{95} \%\right.$. Las variables independientes que se incluyeron en los modelos de regresión de regresión logística fueron: la edad, el sexo y el grado de control de asma de los niños, y la edad, escolaridad, estado civil y clase del cuidador principal de los niños asmáticos.

La pertinencia del análisis factorial se evaluó mediante el test de Kaiser-Meyer-Olkin y el de esfericidad de Bartlett. Para determinar la estructura factorial de la escala abreviada utilizada en el estudio, se hizo un análisis factorial exploratorio de los componentes principales, con una rotación ortogonal de tipo Varimax. La validez interna se determinó mediante el coeficiente alfa de Cronbach (30).

Todas las pruebas estadísticas fueron de dos colas y el nivel de significancia utilizado fue de 0,05. Los datos se analizaron con el paquete estadístico Stata $12.0^{\mathrm{T} M}$ (Stata Corporation, College Station, Texas, USA).

\section{Resultados}

En el presente análisis se incluyeron los datos de 162 pacientes y sus padres o cuidadores. Del total de pacientes incluidos en el estudio, 94 
$(58,0 \%)$ eran de sexo masculino y los restantes $68(42,0 \%)$ de sexo femenino. La mediana (rango intercuartílico) de la edad de los pacientes incluidos en el estudio fue de 6 años (rango, 4 a 9), 74 (45,7\%) entre 1 y 5 años, 79 (48,8 \%) entre 6 y 12 años, y 9 (5,6 \%) entre 13 y 16 años.

En el presente estudio, al utilizar la puntuación total de la escala abreviada para determinar la sobrecarga de los padres o cuidadores, se encontró que 37 $(22,8 \%)$ estaban experimentando una sobrecarga intensa y los restantes 125 (77,2\%) no.

La estadística descriptiva de otras variables relacionadas con características de los niños asmáticos y de sus padres o cuidadores, se muestran en el cuadro 2, agrupados según el nivel de sobrecarga.

El test de Kaiser-Meyer-Olkin $(0,847)$ y el test de esfericidad de Bartlett $(349,5 ; \quad p<0,001)$ mostraron que la muestra cumplía los criterios para el análisis factorial. Se hizo un análisis factorial de componentes principales, con una rotación ortogonal de tipo Varimax. Este mostró una estructura probable de dos factores que, en conjunto, explican el $61,5 \%$ de la varianza total de los resultados. El primer factor incluyó preguntas relacionadas con efectos en la vida personal de los cuidadores, y el segundo factor incluyó preguntas relacionadas con desatención de la propia salud y de las relaciones familiares y sociales (cuadro $3)$. Un tercer factor, con el que se explicaría hasta el $73,3 \%$ de la varianza, no pudo mantenerse en función del criterio de Kaiser por alcanzar un valor propio (Eigenvalue) de 0,82.

La validez interna se determinó mediante el coeficiente alfa de Cronbach. En el cuadro 4 se muestran los valores de este coeficiente para cada una de las preguntas (con un rango entre 0,774 y $0,7932)$ y para el total de la escala $(0,8085)$.

La validez de constructo se valoró comparando el grado de control del asma de los niños con padres o cuidadores con sobrecarga intensa, con el de aquellos con padres o cuidadores con menos sobrecarga. Según lo esperado, una mayor proporción de niños con asma no controlada tenían padres o cuidadores con sobrecarga intensa, en comparación con los que tenían padres o cuidadores sin la misma sobrecarga $(81,1$ Vs. 55,$2 ; p=0,005)$. Igualmente, una mayor proporción de niños con asma controlada tenían padres o cuidadores sin sobrecarga intensa, en comparación con los que tenían padres o cuidadores con sobrecarga intensa $(27,2$ Vs. 8,$1 ; p=0,014)$.

Para determinar los factores asociados con una sobrecarga intensa en los padres o cuidadores, en primer lugar se hizo un análisis bivariado entre cada una de las variables independientes analizadas y la

Cuadro 2. Estadística descriptiva de variables relacionadas con características de los niños asmáticos y sus padres o cuidadores, agrupados según el nivel de sobrecarga

\begin{tabular}{|c|c|c|c|c|}
\hline Variable & $\begin{array}{l}\text { Todos los pacientes y sus } \\
\text { padres o cuidadores } \\
(n=162)\end{array}$ & $\begin{array}{l}\text { Padres o cuidadores con } \\
\text { sobrecarga intensa } \\
(n=37)\end{array}$ & $\begin{array}{l}\text { Padres o cuidadores sin } \\
\text { sobrecarga intensa } \\
\qquad(n=125)\end{array}$ & $\mathbf{p}$ \\
\hline Edad del niño; mediana (RIC) años * & $6,0(4,0-9,0)$ & $6,0(4,0-8,5)$ & $6,0(4,0-9,0)$ & 0,73 \\
\hline Edad del cuidador; mediana (RIC) años & $38,0(32,0-43,2)$ & $39,0(34,0-45,5)$ & $38,0(32,0-43,0)$ & 0,19 \\
\hline Sexo del niño; M/F & $94 / 68$ & $21 / 16$ & $73 / 52$ & 0,86 \\
\hline \multicolumn{5}{|l|}{ Clase de cuidador } \\
\hline Madre & $132(81,5 \%)$ & $31(83,8 \%)$ & $101(80,8 \%)$ & 0,68 \\
\hline Padre & $9(5,6 \%)$ & $0(0,0 \%)$ & $9(7,2 \%)$ & 0,46 \\
\hline Ambos padres & $2(1,2 \%)$ & $0(0,0 \%)$ & $2(1,6 \%)$ & 1,00 \\
\hline Abuelos & $14(8,6 \%)$ & $4(10,8 \%)$ & $10(8,0 \%)$ & 0,52 \\
\hline Otro & $5(3,1 \%)$ & $2(5,4 \%)$ & $3(2,4 \%)$ & 0,32 \\
\hline \multicolumn{5}{|l|}{ Nivel de escolaridad del cuidador } \\
\hline Primaria (completa o incompleta) & $22(13,6 \%)$ & $6(16,2 \%)$ & $16(12,8 \%)$ & 0,59 \\
\hline Bachillerato (completo o incompleto) & $72(44,4 \%)$ & $14(37,8 \%)$ & $58(46,4 \%)$ & 0,36 \\
\hline Técnico o universitario & $68(42,0 \%)$ & $17(45,9 \%)$ & $51(40,8 \%)$ & 0,58 \\
\hline \multicolumn{5}{|l|}{ Estado civil } \\
\hline Casados & $69(42,6 \%)$ & $14(37,8 \%)$ & $55(44,0 \%)$ & 0,51 \\
\hline Solteros & $25(15,4 \%)$ & $5(13,5 \%)$ & $20(16,0 \%)$ & 0,71 \\
\hline Unión libre & $68(42,0 \%)$ & $18(48,6 \%)$ & $50(40,0 \%)$ & 0,35 \\
\hline \multicolumn{5}{|l|}{ Grado de control del asma } \\
\hline No controlada & $99(61,1 \%)$ & $30(81,1 \%)$ & $69(55,2 \%)$ & 0,005 \\
\hline Parcialmente controlada & $26(16,0 \%)$ & $4(10,8 \%)$ & $22(17,6 \%)$ & 0,45 \\
\hline Controlada & $37(22,8 \%)$ & $3(8,1 \%)$ & $34(27,2 \%)$ & 0,014 \\
\hline
\end{tabular}

* RIC: rango intercuartílico 
variable 'resultado'. La única variable que se asoció con una sobrecarga intensa fue el grado de control del asma (cuadro 2). Posteriormente, se ajustaron los modelos de regresión logística para identificar las variables asociadas de forma independiente con la presencia de sobrecarga intensa. La única variable independiente que se asoció significativamente con una sobrecarga intensa en el análisis multivariado, fue el asma no controlada (OR=5,38; $\mathrm{IC}_{95} \%: 1,43$ 20,16; $p=0,012)$. La edad del cuidador estuvo muy cerca de asociarse significativamente con la variable 'resultado' (OR=1,05; $I_{95} \%$ : 0,99-1,11; $p=0,057$ ) (cuadro 5).

\section{Discusión}

En el presente estudio se mostró que la versión abreviada de la escala de Zarit tiene una estructura factorial de dos dominios, una aceptable validez interna y una adecuada validez de constructo para determinar la sobrecarga que experimentan los padres o cuidadores de niños asmáticos. Además,

Cuadro 3. Análisis factorial exploratorio sobre posibles dominios de las preguntas que forman la escala abreviada de Zarit*

\begin{tabular}{lcc}
\hline Pregunta & Factor I & Factor II \\
\hline Valor propio (Eigenvalue) & 3,269 & 1,035 \\
Porcentaje de varianza & $46,71 \%$ & $14,80 \%$ \\
Pregunta 1 & 0,700 & \\
Pregunta 2 & 0,791 & \\
Pregunta 3 & & 0,677 \\
Pregunta 4 & & 0,845 \\
Pregunta 5 & & 0,774 \\
Pregunta 6 & 0,587 & \\
Pregunta 7 & 0,835 & \\
\hline
\end{tabular}

* Factor I: efectos en la vida personal de los cuidadores; factor II: desatención de la propia salud y relaciones familiares y sociales. Se aprecia la existencia de dos probables factores en los que se agrupan las preguntas de la escala, que en conjunto explican el $61,5 \%$ de la varianza total de los resultados.

Cuadro 4. Valores del alfa de Cronbach para cada una de las preguntas que componen la escala abreviada de Zarit utilizada en el estudio y para la escala en conjunto

\begin{tabular}{lc}
\hline Pregunta & Alfa de Cronbach \\
\hline Pregunta 1 & 0,7932 \\
Pregunta 2 & 0,7656 \\
Pregunta 3 & 0,7895 \\
Pregunta 4 & 0,7892 \\
Pregunta 5 & 0,7808 \\
Pregunta 6 & 0,7876 \\
Pregunta 7 & 0,7774 \\
Escala en conjunto & 0,8085 \\
\hline
\end{tabular}

Cuadro 5. Variables asociadas con la presencia de sobrecarga intensa en padres o cuidadores de niños con asma en el análisis multivariado

\begin{tabular}{|c|c|c|}
\hline Variable & $\begin{array}{l}\text { Odds ratio (OR) } \\
\text { (IC95\%) }\end{array}$ & $\mathbf{p}$ \\
\hline Edad del niño & $1,22(0,54-2,74)$ & 0,78 \\
\hline \multicolumn{3}{|l|}{ Sexo del niño } \\
\hline Masculino & $1,22(0,54-2,74)$ & 0,63 \\
\hline Femenino & 1,00 & - \\
\hline \multicolumn{3}{|l|}{ Grado de control de asma } \\
\hline Controlada & 1,00 & - \\
\hline Parcialmente controlada & $2,39(0,47-12,19)$ & 0,29 \\
\hline No controlada & $5,38(1,43-20,16)$ & 0,012 \\
\hline Edad del cuidador & $1,05(0,99-1,11)$ & 0,057 \\
\hline \multicolumn{3}{|l|}{ Estado civil } \\
\hline Casado & 1,00 & - \\
\hline Soltero & $0,78(0,22-2,70)$ & 0,69 \\
\hline Unión libre & $1,98(0,78-5,00)$ & 0,15 \\
\hline \multicolumn{3}{|l|}{ Clase de cuidador } \\
\hline Madre & $2,53(0,62-10,40)$ & 0,20 \\
\hline Otro cuidador & 1,00 & - \\
\hline \multicolumn{3}{|l|}{$\begin{array}{l}\text { Nivel de escolaridad del } \\
\text { cuidador }\end{array}$} \\
\hline Primaria & 1,00 & - \\
\hline Bachillerato & $0,66(0,20-2,15)$ & 0,49 \\
\hline Técnico o universitario & $1,14(0,33-3,88)$ & 0,83 \\
\hline
\end{tabular}

se mostró que el grado de control del asma del niño es la variable independiente más importante de este nivel de sobrecarga, siendo también la edad del cuidador un factor que hay que tener en cuenta.

Estos hallazgos son importantes principalmente por tres aspectos. En primer lugar, pueden ayudar a sensibilizar al cuerpo médico que atiende niños asmáticos y que atiende adultos que tienen niños asmáticos bajo su cuidado, sobre la importancia del síndrome de sobrecarga del cuidador. Debido a que el asma no controlada fue la variable independiente más importante que se asoció con una sobrecarga intensa en los padres o cuidadores y a la alta prevalencia de asma no controlada que se ha reportado en nuestro medio (31), es concebible considerar que el nivel de sobrecarga que experimentan estos padres o cuidadores en nuestro medio es significativo.

Esta sobrecarga, además de los efectos que se han descrito en el cuidador mismo, puede tener importantes repercusiones en la morbilidad por asma de los niños, debido, entre otras cosas, a la mayor prevalencia de trastornos depresivos que se ha descrito en cuidadores con sobrecarga comparados con aquellos sin sobrecarga y con no cuidadores $(8,14)$. La presencia de síntomas depresivos en madres de niños asmáticos se ha asociado con incremento en la morbilidad por 
asma, en el número de consultas al servicio de urgencias y en hospitalizaciones por asma, y en ausencias al trabajo por parte de los padres y al colegio por parte de los niños (32). Además, se ha descrito que las madres con síntomas depresivos suelen tener menores habilidades para tratar a sus hijos con asma y para seguir las instrucciones médicas con respecto al tratamiento, con niveles más bajos de observancia $(33,34)$.

En segundo lugar, la validación inicial de la escala utilizada en el presente estudio sugiere que esta podría ser un instrumento adecuado para medir la sobrecarga que pueden experimentar los padres o cuidadores de niños asmáticos.

En tercer lugar, las variables que se asociaron de forma independiente con la presencia de sobre-carga intensa identificadas en el presente estudio, pueden tener utilidad en la práctica clínica diaria para ayudar a optimizar un proceso de investigación de sobrecarga de padres o cuidadores de niños asmáticos.

Aunque, hasta donde sabemos, el concepto de sobrecarga no se ha medido de forma directa en padres o cuidadores de niños asmáticos, los resultados de otros estudios que han medido calidad de vida (concepto que comprende varios dominios relacionados con el concepto de sobrecarga) en padres o cuidadores de niños asmáticos, están de acuerdo con los hallazgos del presente estudio. AlAkour, et al., (35), y Dalheim-Englund, et al., (36) reportaron niveles más bajos de calidad de vida en padres de niños con asma grave, con respecto a padres de niños con asma leve. De igual forma, Stelmach, et al., reportaron una asociación significativa entre la calidad de vida de los padres de niños asmáticos medida con el Pediatric Asthma Caregiver's Quality of Life Questionnaire (PACQLQ), con el grado de control de asma de sus hijos, encontrando un cambio significativo en la puntuación de dicho cuestionario en los padres de los niños que lograron un adecuado control de su asma (37).

Aunque al determinar la estructura factorial de la escala original también utilizando un análisis factorial exploratorio, Ankri, et al., reportaron la existencia de tres factores (24) y la estructura factorial de la escala abreviada utilizada en este estudio mostró sólo la existencia de dos factores, no parece perderse mucha información con la reducción de la escala, pues el factor denominado "sentimientos de culpa" de la escala original podría ser más aplicable a cuidadores de ancianos con enfermedades degenerativas que a padres 0 cuidadores de niños asmáticos. Sin embargo, para valorar más adecuadamente los tres factores descritos en la escala original en padres 0 cuidadores de niños asmáticos, sería importante hacer un análisis factorial confirmatorio.

Las limitaciones del presente estudio comprenden, en primer lugar, el no haber completado el proceso de validación de la escala que se utilizó. Aunque no se evaluó la validez de apariencia ni la de contenido, porque se consideró que ya habían sido evaluadas adecuadamente en estudios previos, faltó valorar la fiabilidad test-retest y la sensibilidad al cambio, y aportar mayor evidencia sobre la validez de constructo de la escala, valorando la validez convergente (evaluando el grado de acuerdo entre la puntuación de la escala con otra escala que mida el mismo constructo, como por ejemplo, el cuestionario para medir la calidad de vida de padres de niños asmáticos, el PACQLQ). Sin embargo, se considera que el proceso de validación del presente estudio es una aproximación inicial, debiendo completarse este proceso en futuros estudios.

En segundo lugar, en lugar de la escala original, en este estudio se utilizó una versión abreviada. No obstante, esta versión abreviada no sólo ha mostrado disminuir el tiempo para completarla, sino que también ha mostrado tener sensibilidad, especificidad y valores pronóstico adecuados para detectar sobrecarga intensa, al compararse con la escala original. De todos modos, como se mencionó, debido a que se ha reportado que la estructura factorial de la escala original muestra la presencia de tres factores y la de la abreviada que utilizamos en el estudio sólo mostró dos factores, sería importante hacer un análisis factorial confirmatorio de la escala original en padres o cuidadores de niños asmáticos.

En tercer lugar, se hizo un muestreo no probabilístico en una consulta de neumología pediátrica de un hospital de tercer nivel, por lo que es posible que en el estudio se hayan incluido pacientes con un nivel de control más bajo que el promedio de la población general de niños asmáticos, presentando, por lo tanto, sus padres o cuidadores una mayor sobrecarga que el promedio de la población, lo que podría comprometer la validez externa de los resultados.

En cuarto lugar, no se hizo un cálculo formal del tamaño de muestra, lo que podría haber favorecido que el estudio tenga un bajo poder para detectar asociaciones, y explicar, por ejemplo, la falta de 
significancia estadística de la edad del cuidador como variable asociada de forma independiente con la presencia de sobrecarga intensa en el análisis multivariado. Sin embargo, este se puede considerar como un estudio inicial sobre el tema y se requieren estudios posteriores no sólo en una consulta especializada de un hospital de referencia, sino también, incluyendo un mayor número de pacientes.

Por último, en lo que se refiere a la identificación de variables asociadas de forma independiente con la presencia de sobrecarga intensa, al igual que ocurre con todos los estudios observacionales, no se puede excluir la presencia de confusión residual, por lo que los resultados deben interpretarse con cautela.

La principal fortaleza del estudio es el haber hecho el proceso de validación inicial de una escala útil para medir un fenómeno que puede tener importantes repercusiones en la morbilidad y en el uso de recursos de salud por asma en niños, y que no ha sido reportado con anterioridad en la literatura científica en cuidadores de niños asmáticos. Además, se identifican variables que se asocian de forma independiente con sobrecarga intensa en padres o cuidadores de niños asmáticos, lo cual puede ayudar a optimizar un proceso de investigación de este fenómeno.

En resumen, en el presente estudio se mostró que la versión abreviada de la escala de Zarit utilizada tiene una aceptable validez interna y una adecuada validez de constructo para determinar el nivel de sobrecarga que experimentan los padres o cuidadores de niños asmáticos. Además, se demostró que el grado de control del asma del niño es la variable independiente más importante que se asocia con esta sobrecarga, siendo también la edad del cuidador un factor que hay que tener en cuenta.

Consideramos que se requieren estudios adicionales para completar el proceso de validación de la escala, hacer un análisis factorial confirmatorio de la escala original en padres o cuidadores de niños asmáticos para verificar la estructura factorial descrita de dicha escala, y futuros estudios para identificar los factores predictores de sobrecarga intensa en otros ámbitos diferentes a un servicio especializado de neumología pediátrica de un hospital de tercer nivel, e incluyendo un mayor número de pacientes.

Además, es importante diseñar estudios futuros que tengan por objeto determinar si las intervenciones dirigidas a disminuir la sobrecarga en padres o cuidadores de niños asmáticos son eficaces para mejorar resultados clínicamente importantes en niños asmáticos.

\section{Agradecimientos}

Los autores agradecen a Mónica Patricia Sossa, por su colaboración con el análisis estadístico y la revisión del manuscrito.

\section{Conflicto de intereses}

Los autores declaran no tener ningún tipo de conflicto de interés.

\section{Financiación}

El presente estudio fue financiado parcialmente por el Laboratorio Farmacéutico Novartis de Colombia, S. A.

\section{Referencias}

1. Caraballo L, Cadavid A, Mendoza J. Prevalence of asthma in a tropical city of Colombia. Ann Allergy. 1992;68:525-9.

2. Dennis R, Caraballo L, García E, Caballero A, Aristizábal G, Córdoba $\mathbf{H}$, et al. Asthma and other allergic conditions in Colombia: A study in 6 cities. Ann Allergy Asthma Immunol. 2004;93:568-74. http://dx.doi.org/10.1016/S10811206(10)61265-3

3. García E, Aristizábal G, Vásquez C, Rodríguez-Martínez CE, Sarmiento OL, Satizabal CL. Prevalence of and factors associated with current asthma symptoms in school children aged 6-7 and 13-14 yr old in Bogotá, Colombia. Pediatr Allergy Immunol. 2008;19:307-14. http://dx.doi. org/10.1111/j.1399-3038.2007.00650.x

4. Dennis R, Caraballo L, García E, Rojas MX, Rondón MA, Pérez A, et al. Prevalence of asthma and other allergic conditions in Colombia 2009-2010: A cross-sectional study. BMC Pulmonary Medicine. 2012,12:17. http://dx.doi. org/10.1186/1471-2466-12-17.

5. Juniper EF, Guyatt, GH, Feeny PJ, Ferrie LE, Townsend M. Measuring quality of life in the parents of children with asthma. Qual Life Res. 1996;5:27-34.

6. Zarit SH, Reever KE, Bach-Peterson J. Relatives of the impaired elderly: Correlates of feelings of burden. Gerontologist. 1980;20:649-55.

7. Seguí JD, Ortiz-Tallo M, De Diego Y. Factores asociados al estrés del cuidador primario de niños con autismo: sobrecarga, psicopatología y estado de salud. Anales de Psicología. 2008;24:100-5.

8. Thompson CCA, Spilsbury K. Support for carers of people with Alzheimer's type dementia. Cochrane Database of Systematic Reviews 1998, Issue 3. Art. No.: CD000454. http://dx.doi.org/10.1002/14651858.CD000454

9. Marriot A, Donaldson C, Terrier N, Burns A. Effectiveness of cognitive-behavioural family intervention in reducing the burden of care in carers of patients with Alzheimer's disease. Br J Psychiatry. 2000;176:557-62. http://dx.doi.org/10.1192/ bjp.176.6.557 
10. Mittelman MS, Ferris SH, Shulman E, Steinberg G, Levin B. A family intervention to delay nursing home placement of patients with Alzheimer disease. A randomized controlled trial. JAMA. 1996;276:1725-31. http://dx.doi.org/10.1001/ jama.1996.03540210033030.

11. Wisniewski S, Belle SH, Coon DW, Marcus SM, Ory MG, Burgio LD, et al. The Resources for Enhancing Alzheimer's Caregiver Health (REACH): Project design and baseline characteristics. Psychol Aging. 2003;18:375-84. http:// dx.doi.org/1010.1037/0882-7974.18.3.375

12. López O, Lorenzo A. Morbilidad en cuidadores de pacientes confinados en su domicilio. Aten Primaria. 1999;24:404-10.

13. Schulz R, Beach S. Caregiving as a risk factor for mortality. The caregiver health effects study. JAMA. 1999;282:221519. http://dx.doi.org/10.1001/jama.282.23.2215.

14. Pinquart M, Sörensen S. Differences between caregivers and noncaregivers in psychological health and physical health: A meta-analysis. Psychol Aging 2003;18:250-67. http://dx.doi.org/1010.1037/0882-7974.18.2.250

15. Bristol M. Family resources and successful adaptation to autistic children. New York: Plenum Press; 1984. p. 289310.

16. Beltrán FJ, Torres IA, Vásquez F, Barrientos C, Lin D, Beltrán AA, et al. Calidad de vida en cuidadores de enfermos asmáticos: un estudio comparativo. Psicología y Salud. 2008;18:181-7.

17. Erickson SR, Munzenberger PJ, Plante MJ, Kirking DM, Hurwitz ME, Vanuya RZ. Influence of sociodemographics on the health-related quality of life of pediatric patients with asthma and their caregivers. J Asthma. 2002;39:107-17.

18. Vidal A, Duffau G, Ubilla C. Calidad de vida en el niño asmático y su cuidador. Rev Chil Enferm Respir. 2007;23:160-6. 73482007000300002

19. Murphy KR, Fitzpatrick S, Cruz-Rivera M, Miller CJ, Parasuraman B. Effects of budesonide inhalation suspension compared with cromolyn sodium nebulizer solution on health status and caregiver quality of life in childhood asthma. Pediatrics. 2003;112:212-9.

20. Juniper EF. Health-related quality of life in asthma. Curr Opin Pulm Med. 1999;5:105-10.

21. Gort M, March J, Gómez X, de Miguel M, Mazarico S, Ballesté J. Escala de Zarit reducida en cuidados paliativos. Med Clin (Barc). 2005;124:651-3.

22. Global Strategy for Asthma Management and Prevention, Global Initiative for Asthma (GINA) 2011. Fecha de consulta: 27 de julio 27 de 2012. Disponible en: http://www. ginasthma. org/

23. Martín M, Salvadó I, Nadal S, Miji LC, Rico JM, Lanz P. Adaptación para nuestro medio de la escala de sobrecarga del cuidador (Caregiver Burden Interview) de Zarit. Rev Gerontol. 1996;6:338-46.
24. Ankri J, Andrieu S, Beaufils B, Grand A, Henrard J. Beyond the global score of the Zarit Burden Interview: Useful dimensions for clinicians. Int J Geriatr Psychiatry. 2005;20:254-60. http://dx.doi.org/1010.1002/gps.1275

25. Vélez JM, Berbesi D, Cardona D, Segura A, Ordóñez J. Validación de escalas abreviadas de Zarit para la medición de síndrome del cuidador primario del adulto mayor en Medellín. Aten Primaria. 2012;44:411-6.

26. Arai Y, Kudo K, Hosokawa T, Washio M, Miura H, Hisamichi S. Reliability and validity of the Japanese version of the Zarit Caregiver Burden interview. Psychiatry Clin Neurosci. 1997;51:281-7.

27. Herbert R, Bravo G, Preville M. Reliability, validity and reference values of the Zarit Burden Interview for assessing informal caregivers of community-dwelling older persons with dementia. Can J Aging 2000;19:494-507. http://dx.doi. org/10.1017/S0714980800012484

28. Taub A, Andreoli SB, Bertolucci PH. Dementia caregiver burden:Reliability of the Brazilianversion of the Zaritcaregiver burden interview. Cad Saúde Pública. 2004;20:372-6. http:// dx.doi.org/10.1590/S0102-311X2004000200004

29. Regueiro AA, Pérez A, Gómara S, Ferreriro M. Escala de Zarit reducida para la sobrecarga del cuidador en atención primaria. Aten Primaria. 2007;39:185-8.

30. Cronbach LJ. Coefficient alpha and the internal structure of test. Psychometrika. 1951;16:297-333.

31. Neffen H, Fritscher C, Cuevas F, Levy G, Chiarella P, Soriano JB, et al. Asthma control in Latin America: The Asthma Insights and Reality in Latin America (AIRLA) survey. Rev Panam Salud Pública. 2005;17:191-7.

32. Pak L, Allen PJ. The impact of maternal depression on children with asthma. Pediatr Nurs. 2012;38:11-9.

33. Shalowitz MU, Berry CA, Quinn KA, Wolf RL. The relationship of life stressors and maternal depression to pediatric asthma morbidity in a subspecialty practice. Ambul Pediatr. 2001;1:185-93.

34. Bartlett SJ, Krishnan JA, Riekert KA, Butz AM, Malveaux FJ, Rand CS. Maternal depressive symptoms and adherence to therapy in inner-city children with asthma. Pediatrics. 2004;113:229-37. http://dx.doi.org/10.1542/ peds.113.2.229

35. Al-Akour N, Khader YS. Having a child with asthma-quality of life for Jordanian parents. Int J Nurs Pract. 2009;15:574-9. http://dx.doi.org/10.1111/j.1440-172X.2009.01796.x.

36. Dalheim-Englund AC, Rydström I, Rasmussen BH, Möller C, Sandman PO. Having a child with asthma-quality of life for Swedish parents. J Clin Nurs. 2004;13:386-95. http://dx.doi.org/1010.1046/j.1365-2702.2003.00825.x

37. Stelmach I, Podlecka D, Smejda K, Majak P, Jerzy $\square$ ska J, Stelmach R, et al. Pediatric Asthma Caregiver's Quality of Life Questionnaire is a useful tool for monitoring asthma in children. Qual Life Res 2012;21:1639-42. http://dx.doi. org/10.1007/s11136-011-0070-x 